\title{
En mayores de 60 años el tabaquismo activo duplica la mortalidad cardiovascular y su cesación la reduce
}

In adults over 60 years, active smoking doubles the risk of cardiovascular mortality and quitting reduces it

Ute Mons y cols. BMJ 2015;350:h1551

\section{Objetivos}

Establecer el impacto del tabaquismo activo y del cese tabáquico en la mortalidad cardiovascular, eventos coronarios agudos, y accidentes cerebrovasculares (ACV) en personas mayores de 60 años.

\section{Diseño}

Meta-análisis de datos individuales, que utilizó información de 25 cohortes participantes del consorcio CHANCES, ELSA y NHANES III.

\section{Población y lugar}

Se incluyeron en el estudio 503.905 participantes mayores de 60 años, reclutados en Europa y América del Norte, con un período de seguimiento de 8 a 13 años.

\section{Mediciones}

Resultado principal: eventos cardiovasculares fatales, obtenidos mediante certificados de defunción de acuerdo a los códigos de la Clasificación Internacional de Enfermedades. Resultados secundarios: incidencia de eventos coronarios agudos (eventos fatales y no fatales de infarto agudo de miocardio, angina inestable o muerte coronaria); e incidencia de ACV (eventos fatales y no fatales que satisfacían los signos y síntomas típicos de hemorragia subaracnoidea, hemorragia intracerebral o infarto cerebral). El status de tabaquismo y su intensidad se evaluó utilizando entrevistas o cuestionarios autoadministrados. Se establecieron categorías para el estado actual de tabaquismo (fumador, exfumador, no fumador), intensidad (<10 cigarrillos al día, 10 a 19 cigarrillos al día, $\geq 20$ cigarrillos al día) y antigüedad del cese tabáquico ( $<5$ años atrás, 5 a 9 años atrás, 10 a 19 años atrás y $\geq 20$ años atrás).

\section{Resultados principales}

Los resultados de los fumadores respecto de los no fumadores se muestran en la tabla 1. En la tabla 2 se muestran los de los ex-fumadores respecto de los fumadores.

Tabla 1: Riesgo cardiovascular en fumadores respecto de no fumadores.

\begin{tabular}{l|c|c|c|c}
\multirow{2}{*}{$\begin{array}{c}\text { Exposición al } \\
\text { tabaco }\end{array}$} & \multicolumn{2}{|c|}{ Mortalidad Cardiovascular } & Eventos Coronarios Agudos & ACV \\
\cline { 2 - 5 } & HR (IC 95\%) & RAP* (IC95\%) & HR (IC 95\%) & HR (IC 95\%) \\
\hline Total & $2,07(1,82 \mathrm{a} 2,36)$ & $5,50(4,25 \mathrm{a} 6,75)$ & $1,98(1,75 \mathrm{a} 2,25)$ & $1,58(1,40 \mathrm{a} 1,78)$ \\
\hline $\mathbf{1 0}$ cigarrillos al día & $1,87(1,63 \mathrm{a} 2,15)$ & $4,46(3,33 \mathrm{a} 5,59)$ & $1,74(1,52 \mathrm{a} 2,01)$ & $1,43(1,24 \mathrm{a} 1,64)$ \\
\hline $\mathbf{1 0}$ a $\mathbf{1 9}$ cigarrillos al día & $1,94(1,65 \mathrm{a} 2,28)$ & $4,80(3,34 \mathrm{a} 6,27)$ & $1,83(1,56 \mathrm{a} 2,15)$ & $1,60(1,41 \mathrm{a} 1,82)$ \\
\hline$\geq \mathbf{2 0}$ cigarrillos al día & $2,63(2,28 \mathrm{a} 3,04)$ & $6,90(5,59 \mathrm{a} 8,20)$ & $2,43(2,01 \mathrm{a} 2,93)$ & $1,91(1,66 \mathrm{a} 2,21)$ \\
\hline
\end{tabular}

*RAP: Risk Advancement Period Representa la cantidad de tiempo en años en el que un evento se adelanta en individuos expuestos en comparación con individuos no expuestos. HR: hazard ratio. IC 95\%: intervalo de confianza del 95\%.

Tabla 2: Riesgo cardiovascular en exfumadores respecto de fumadores.

\begin{tabular}{l|c|c|c|c}
\multirow{2}{*}{$\begin{array}{c}\text { Tiempo de } \\
\text { cesación }\end{array}$} & \multicolumn{2}{|c|}{ Mortalidad Cardiovascular } & Eventos Coronarios Agudos & ACV \\
\cline { 2 - 5 } & HR (IC 95\%) & RAP* (IC95\%) & HR (IC 95\%) & HR (IC 95\%) \\
\hline Cese $<\mathbf{5}$ años atrás & $0,90(0,81 \mathrm{a} 1,00)$ & $-0,82(-1,72 \mathrm{a} 0,07)$ & $0,84(0,72 \mathrm{a} 0,98)$ & $0,97(0,79 \mathrm{a} 1,19)$ \\
\hline Cese 5-9 años atrás & $0,84(0,73 \mathrm{a} 0,95)$ & $-1,34(-2,29 \mathrm{a}-0,39)$ & $0,86(0,72 \mathrm{a} 1,02)$ & $0,98(0,74 \mathrm{a} 1,31)$ \\
\hline Cese $\mathbf{1 0 - 1 9}$ años atrás & $0,78(0,71 \mathrm{a} 0,85)$ & $-1,96(-2,69 \mathrm{a}-1,24)$ & $0,69(0,58 \mathrm{a} 0,82)$ & $0,79(0,69 \mathrm{a} 0,92)$ \\
\hline Cese $\geq \mathbf{2 0}$ años atrás & $0,61(0,54 \mathrm{a} 0,69)$ & $-3,94(-4,86 \mathrm{a}-3,03)$ & $0,58(0,46 \mathrm{a} 0,72)$ & $0,67(0,60 \mathrm{a} 0,76)$ \\
\hline
\end{tabular}

*RAP: Risk Advancement Period. Representa la cantidad de tiempo en años en el que un evento se adelanta en individuos expuestos en comparación con individuos no expuestos. HR: hazard ratio. IC 95\%: intervalo de confianza del 95\%.

\section{Conclusión}

El estudio corrobora y expande la evidencia de que el tabaquismo es un importante factor de riesgo independiente de eventos cardiovasculares y mortalidad, incluso a edades avanzadas, duplicando el riesgo y adelantando la mortalidad cardiovascular más de cinco años. A su vez, demuestra que el cese tabáquico en estos grupos etarios sigue siendo beneficioso al reducir el exceso de riesgo, siendo este beneficio mayor a medida que aumenta el tiempo de cese.

Fuentes de financiamiento/conflictos de interés: Los autores declaran no tener conflictos de interés respecto de este estudio.

\section{Comentario}

La incidencia de enfermedad cardiovascular aumenta con la edad y la mayoría de los eventos ocurren en adultos mayores'. El envejecimiento general de la población y el aumento de la expectativa de vida a nivel mundial nos debe llevar a tomar medidas para reducir el riesgo cardiovascular en este grupo. La Argentina presenta una alta prevalencia de tabaquismo: uno de cada cuatro personas es fumadora ${ }^{2}$. Si bien mucha evidencia coloca al tabaquismo como un importante factor de riesgo cardiovascular modificable, existen pocos estudios enfocados específicamente en la población de mayor edad.
Este meta-análisis aporta evidencia para sostener que el cese tabáquico es beneficioso incluso luego de los 60 años, y que el tabaquismo constituye a esa edad un factor de riesgo independiente con un elevado peso sobre la mortalidad cardiovascular.

Conclusiones del comentador

Debemos continuar realizando intervenciones para lograr el cese tabáquico incluso en los pacientes mayores, derribando la idea que poseen muchas personas de que "ya es demasiado tarde".

Alan Gabriel Gauna [ Servicio de Medicina Familiar y Comunitaria, Hospital Italiano de Buenos Aires. alan.gauna @ hospitalitaliano.org.ar]

Gauna AG. En mayores de 60 años el tabaquismo activo duplica la mortalidad cardiovascular y su cesación la reduce. Evid Act Pract Ambul. 2016;19(3):84. Comentado de: Mons U, y col. Impact of smoking and smoking cessation on cardiovascular events and mortality among older adults: meta-analysis of individual participant data from prospective cohort studies of the CHANCES consortium. BMJ. 2015;350:h1551. PMID: 25896935.

\section{Referencias}

1. Kriekard P, y col Primary and secondary prevention of cardiovascular disease in older adults: a status report. Clin Geriatr Med 2009;25:745-55.

2. Instituto Nacional De Estadísticas y Censos (INDEC). $3^{\circ}$ Encuesta Nacional de factores de Riesgo (ENFR). Argentina; 2013. 